\title{
OGSR: A Low Complexity Galileo Software Receiver using Orthogonal Data and Pilot Channels
}

\author{
Ali Albu-Rghaif, Ihsan A. Lami, Maher Al-Aboodi
}

\begin{abstract}
To improve localisation accuracy and multipath rejection, the Galileo-OS signal offers a new modulation with efficient power distribution technique between the two data and pilot navigation components. To achieve the full benefits of this modulation, a robust acquisition and tracking methods must be deployed. For example, using two parallel correlation channels to acquire these data and pilot will gain $3 \mathrm{~dB}$ over using a single channel acquisition correlating with either one of them. However, dual channel SW receivers cost more processing overheads. In this paper, the authors propose to orthogonalise the received data and pilot signals so to enable their acquisition in a single correlation channel bandpass sampling receiver. Our simulation results, using Simulink, prove that OGSR performance is maintained (preserving the $3 \mathrm{~dB}$ gain) with less processing time while the implementation complexity is reduced by $50 \%$.
\end{abstract}

Keywords-Composite BOC; Galileo Acquisition; Orthogonaldata-pilot;

\section{Introduction}

The European Galileo system provides high localisation accuracy and is interoperable with other GNSS signals, such as GPS and GLONASS signals. The Galileo-E1 open service (OS) signal consists of two channels, and each channel has different components. The first one is called data channel (B) that comprises the navigation message, data's primary code and subcarrier frequency. The second one is named pilot channel (C) that includes two pilot's codes (primary and secondary) and subcarrier frequency. In addition, the power of the Galileo-OS signal has been designed with 50/50 power split between the pilot and data channels [1]. These channels are combined and shipped simultaneously at $1575.42 \mathrm{MHZ}$ carrier. Hence, it is possible to shortcut the receiver's acquisition process by acquiring only the pilot or only the data signal using a single correlation chain. However, this leads to a $3 \mathrm{~dB}$ power loss which is important when being acquired in harsh environments. Consequently, dual-channel (DC) acquisition is preferable to enhance the probability of detection of weaker signals. Obviously, the hardware implementation of the dual-channel acquisition will require double the size/resources while the software implementation will require more processing time as well as the resources overheads.

\footnotetext{
Ali Albu-Rghaif, Ihsan Lami, Maher Al-Aboodi

Department of Applied Computing

University of Buckingham

UK
}

OGSR implementation overcomes the dual-correlation channels complexity by combining the data and the pilot signals in a single orthogonal format. This is accomplished by shifting the phase a copy of the received signals by 90 -degrees and then adding it to the original received signal. The motivation of having an orthogonal signal is to be able to perform the acquisition in a single correlation chain, yet offering the same performance as using two correlation chains. OGSR implementation will require, as an overhead, having an orthogonally generated signal instead of having data and pilot generated signals separately. Note that the phase shift of data and pilot signals is equal, and the newly formed orthogonal signal will have the same power as the received signal.

The advantages of OGSR are:

1- Reduces the cost of the acquisition process by saving valuable resources, such as mixing and transforming operations.

2- Maintains the $3 \mathrm{~dB}$ power gain of the received two signals, only in an orthogonal format.

3- Decreases the acquisition time, which will accelerate the TTFF.

4- Provides a cost effective implementation algorithm for Smartphone's software receiver.

OGSR's performance has been analysed based on the simulation environment using MATLAB-Simulink platform. The results show that OGSR's processing time is equal to a single-channel's (SC) processing time. Also, it performs as good as the DC acquisition method but with less number of processing resources.

The rest of the paper is organized as follows: Section II summarises the literature survey on previous joint data and pilot acquisition methods. Section III details the mathematical representation of the Galileo-OS signal while Section IV describes the implementation of our OGSR method. Section V shows the results of the OGSR's performance when compared with other dual-channel and single-channel acquisition methods, and Section VI concludes the OGSR's implementation.

\section{Literature Survey}

A joint-data-pilot signal acquisition method is necessary to acquire Galileo-OS signal so to gain the benefits of this transmission. Therefore, Galileo-OS signal acquisition needs at least two correlation channels to perform the joining between the received data and the pilot signals. A typical acquisition process, in time domain, is where the received Galileo signal is multiplied by two orthogonal carrier 
frequencies, and then the output of the in-phase and the quadrature-phase components will go through two branches, as shown in Figure 1. The two branches are then correlated with the data channel components in the first branch and the pilot channels components in the second branch. The detecting stage is accomplished by joining the output from these two branches, resulting in a $3 \mathrm{~dB}$ gain as well as overcoming the ambiguity between the navigation message in the data channel and the secondary code in the pilot channel. However, this implementation requires four correlation channels [2].

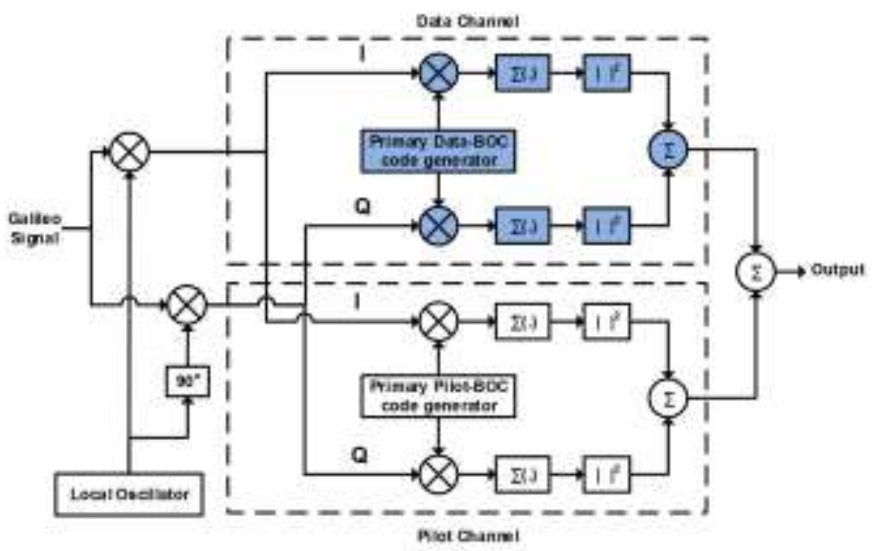

Figure 1 DC acquisition method based serial search

To implement a frequency domain acquisition (FFT-search algorithm) for the Galileo-OS signal, solutions either acquire the data or the pilot signal alone, i.e. single channel acquisition (SC), or still join the data and pilot signals, but using less correlation channels than a time-domain implementation. Figure 2 shows an example of a joint data-pilot channels implementation. This solution claims that combining the powers of the two data and pilot signals provide around $2.8 \mathrm{~dB}$ improvement to the signal detection performance over the acquisition of the data or pilot signal alone. However, this implementation does require two correlation channels [3].

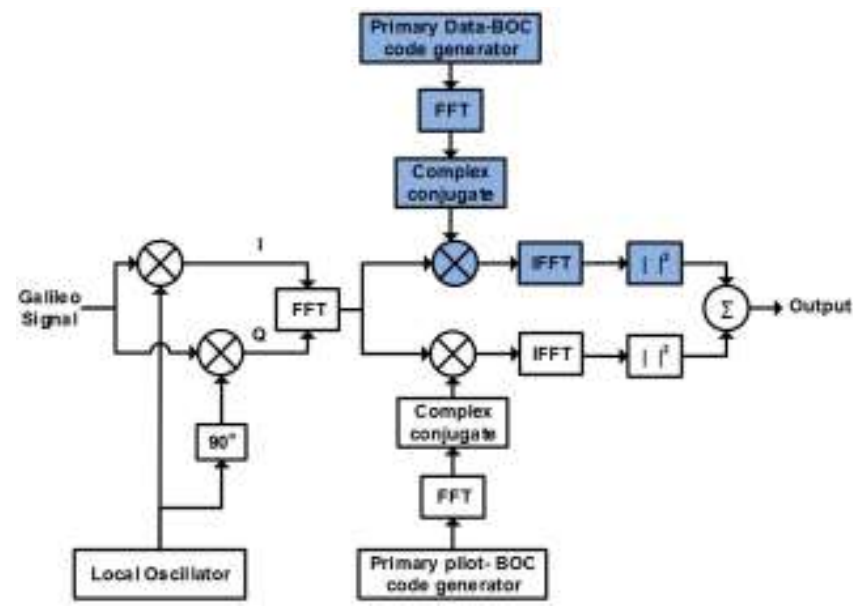

Figure 2 DC acquisition method based FFT search

A further enhancement has been achieved by a differential joint-data-pilot acquisition solution (DfDC) [4]. In this implementation, the complex correlator output of each branch is multiplied by the delayed copy of itself, as shown in Figure
3. Where, the two branches are the correlation of the powerdifference between data and pilot signals (B-C) and the powersum of the data and pilot signals $(B+C)$. These multiplication outputs are then summed up to complete this differential joining process. This solution claims that this post-processing would help to improve the acquisition of the low power signals that has $\mathrm{C} / \mathrm{N}<27 \mathrm{~dB}-\mathrm{Hz}$ by $2 \mathrm{~dB}$. The performance of this work was compared with other SC and DC acquisition methods; their results showed that the differential acquisition has better performance, but obviously at the expense of higher complexity.

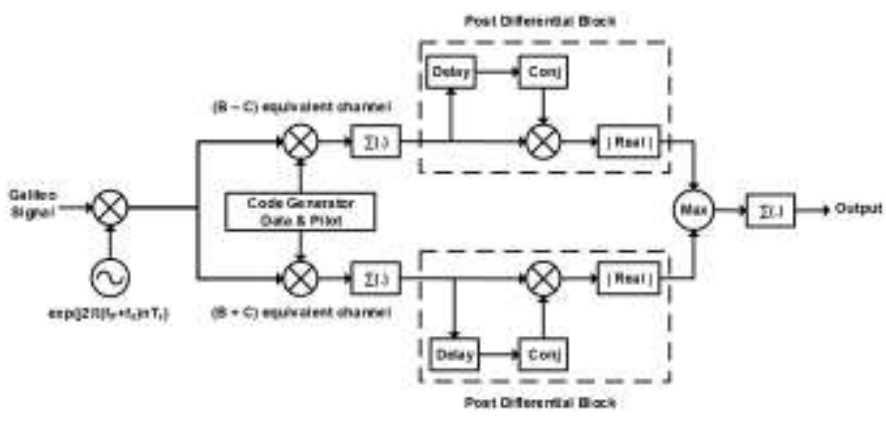

Figure 3 DfDC acquisition Method

In the same vein, a space differential acquisition (SDfC) solution claims to save half of the memory requirements used in the DfDC solution. This achieved by using the phases of both data and pilot signals at the same time (rather than one at a time as used in DfDC) because the data and the pilot signals are transmitted simultaneously by the same source, which means the Doppler and phase shifts of both signals are the same. As depicted in Figure 4, the complex correlators' outputs are separated into the In-phase and the Quadraturephase parts. Then the acquisition process is accomplished by summing the multiplication outputs of the real parts (I_data $X$ I_pilot) and the imaginary parts (Q_data $x$ Q_pilot). This solution, therefore, has better detection probability than the DC solution by around $2 \mathrm{~dB}$, and it is better than the $\mathrm{SC}$ solution by $5 \mathrm{~dB}$. However, it requires more computational operations to split the real and imaginary components of both signals, as well as requiring more resources, such as mixers in time domain, to multiply the complex correlator outputs [5].

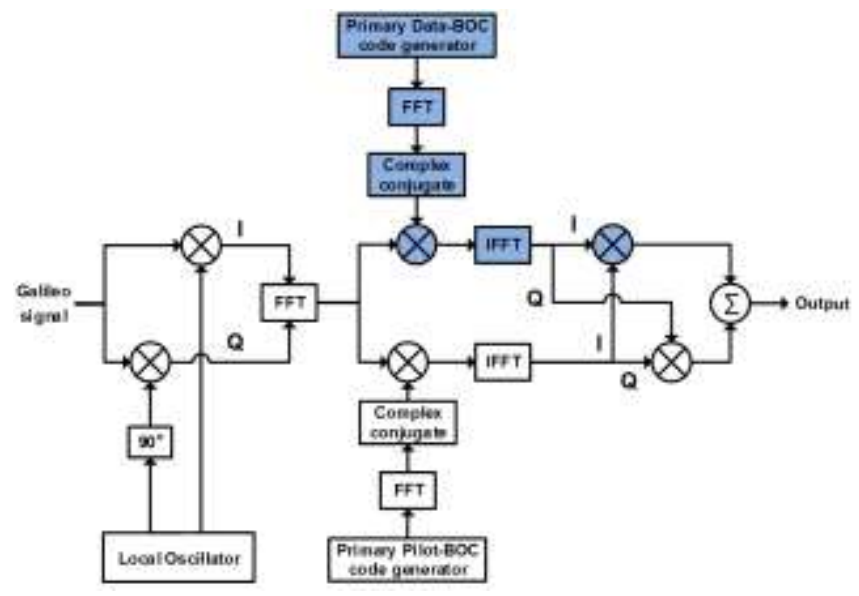

Figure 4 SDfC acquisition Method 
To save valuable correlation resources, orthogonalising any two BPSK signals in a bandpass sampling receiver have been successfully implemented to track two signals simultaneously [6]. In this implementation, one of the received signals was passed through a Hilbert Transform (HT) and then combined with the other signal to construct an orthogonal signal. The orthogonal signal is then folded to the same reference frequency in the First Nyquist Zone by using a single analogue to digital converter. It is worthwhile to mention that this orthogonality has been implemented in the RF front-end to simplify the digital processing.

Our proposed OGSR in this paper capitalise on this idea to design an orthogonal acquisition chain for the Galileo-OS data and pilot signals, thus saving valuable resources and processing time.

\section{The Mathematical Model of the Galileo-OS Signal}

The Galileo-E1-OS signal uses the Composite-BOC (CBOC) modulation, which means multi-level spreading symbols formed from the weighted sum of $\mathrm{BOC}(1,1)$ and $\mathrm{BOC}(6,1)$ as expressed in the following equations [7].

$$
\begin{aligned}
& \mathrm{g}_{\text {BOC }(1,1)}=\left\{\begin{array}{cc}
\operatorname{sign}\left(\sin \left(\frac{2 \pi t}{\mathrm{~T}_{\mathrm{c}}}\right)\right) & 0 \leq \mathrm{t} \geq \mathrm{T}_{\mathrm{c}} \\
\text { and } & \text { elsewhere }
\end{array}\right. \\
& \operatorname{g~}_{\text {вос }(6,1)}=\left\{\begin{array}{cc}
\operatorname{sign}\left(\sin \left(\frac{12 \pi \mathrm{t}}{\mathrm{T}_{\mathrm{c}}}\right)\right) & 0 \leq \mathrm{t} \geq \mathrm{T}_{\mathrm{c}} \\
0 & \text { elsewhere }
\end{array}\right.
\end{aligned}
$$

Where $\mathrm{g}_{\mathrm{BOC}(1,1)}$ represents the $\mathrm{BOC}(1,1)$ spreading symbols, $\mathrm{g}_{\mathrm{BOC}(6,1)}$ represents the $\mathrm{BOC}(6,1)$ spreading symbols, and $\mathrm{T}_{\mathrm{c}}$ is the code chip duration.

This modulation type is used for both the data channel $\left(\mathrm{S}_{\mathrm{b}}\right)$ and for the pilot channel $\left(\mathrm{S}_{\mathrm{C}}\right)$ as expressed in equations (3) and (4) respectively.

$$
\begin{aligned}
& \mathrm{S}_{\mathrm{b}}=\alpha \mathrm{g}_{\text {вос }(1,1)}+\beta \mathrm{g}_{\text {вос }(6,1)} \\
& \mathrm{S}_{\mathrm{c}}=\alpha \mathrm{g}_{\text {вос }(1,1)}-\beta \mathrm{g}_{\text {вос }(6,1)}
\end{aligned}
$$

where $\alpha$ and $\beta$ are the power parameter to control the combined power of the data and pilot channels and are equal to $\sqrt{10 / 11}, \sqrt{1 / 11}$ respectively.

The mathematical representation of the transmitted CBOC signal is shown in equation (5) as described in the Galileo Signal-In-Space Interface Control Document (SIS-ICD) [1].

$\mathrm{X}_{\mathrm{E} 1}=\frac{1}{\sqrt{2}}\left[\mathrm{E}_{\mathrm{B}}(\mathrm{t}) \mathrm{S}_{\mathrm{b}}-\mathrm{E}_{\mathrm{C}}(\mathrm{t}) \mathrm{S}_{\mathrm{c}}\right] \cos \left(2 \pi \mathrm{f}_{\mathrm{E} 1} \mathrm{t}\right)$

where $\mathrm{X}_{\mathrm{E} 1}$ represents the $\mathrm{CBOC}$ Galileo $\mathrm{E} 1$ signal, the $E_{B}$ and $E_{C}$ are the binary signal components (the navigation message and primary/secondary codes) and $\mathrm{f}_{\mathrm{E} 1}$ is the carrier frequency of the $\mathrm{E} 1$ signal at $1.57542 \mathrm{GHz}$.

The CBOC Galileo signal received at the user end is therefore represented as:

$$
\begin{aligned}
\mathrm{X}_{\mathrm{r}}= & \mathrm{A}\left[\mathrm{E}_{\mathrm{B}}\left(\mathrm{n} T_{s}-\tau\right) \mathrm{S}_{\mathrm{b}}-\mathrm{E}_{\mathrm{C}}\left(\mathrm{n} T_{s}-\tau\right) \mathrm{S}_{\mathrm{c}}\right] \\
& e^{\mathrm{j}\left(2 \pi\left(\mathrm{f}_{\mathrm{E} 1}+f_{d}\right) \mathrm{n} T_{s}\right)}+n_{o}\left(\mathrm{n} T_{s}\right)
\end{aligned}
$$

where $X_{r}, A, n, T_{s}, \tau, f$ and $n_{o}$ represent the received signal, the amplitude of the received signal, number of samples, sampling period, code phase delay, carrier frequency, Doppler frequency shift and additive white Gaussian noise respectively.

\section{OGSR Implementation}

OGSR is designed to overcome the complexity of other DC-receivers implementations by half, or in another word, to reduce the overall acquisition/processing time. As shown in Figure 5, this is achieved by making the received Galileo signal orthogonal with a 90-degrees phase-shifted copy of itself, using an HT to do the phase-shift. OGSR is possible only because the Galileo data and pilot signals are transmitted simultaneously, and therefore they have the same Doppler and phase shift as shown in equation (6). Consequently, the 90degrees shift can be applied to any one of the locally generated channel. So, we have chosen the pilot signal to be the phaseshifted signal.

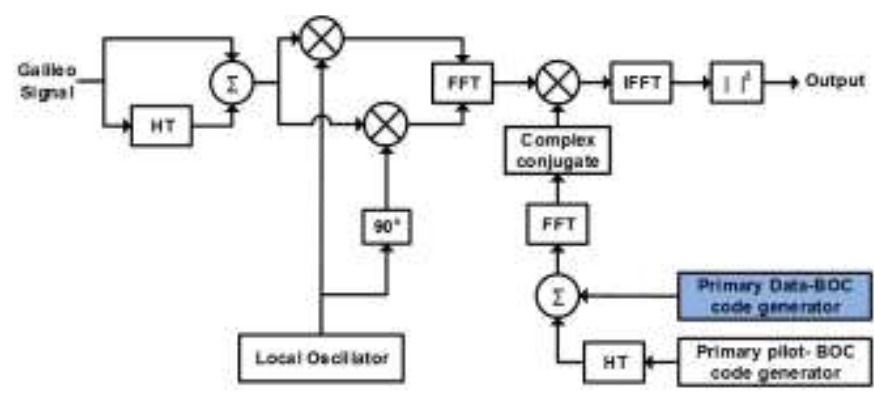

Figure 5. OGSR acquisition method

The following steps illustrate the design process of the OGSR acquisition block. As shown in Figure 5 the resultant combined orthogonal signal is illustrated in equation (7).

$\mathrm{X}_{\mathrm{C}}\left[n T_{s}\right]=\mathrm{X}_{\mathrm{r}}\left[n T_{s}\right]-\mathrm{j} \mathrm{X}_{\mathrm{r}}\left[n T_{s}\right]$

where $\mathrm{X}_{\mathrm{C}}$ represents the complex received signal.

By substituting equation 6 in equation 7 , then this orthogonal signal is:

$\mathrm{X}_{\mathrm{C}}\left[n T_{s}\right]=$

$\left(\mathrm{A}\left[\mathrm{E}_{\mathrm{B}}\left(\mathrm{n} T_{s}-\tau\right) \mathrm{S}_{\mathrm{b}}-\mathrm{E}_{\mathrm{C}}\left(\mathrm{n} T_{s}-\tau\right) \mathrm{S}_{\mathrm{c}}\right] e^{\mathrm{j}\left(2 \pi\left(\mathrm{f}_{\mathrm{E} 1}+f_{d}\right) \mathrm{n} T_{s}\right)}+\right.$

$\left.n_{o}\left(\mathrm{n} T_{s}\right)\right)-$

$\mathrm{j}\left(\mathrm{A}\left[\mathrm{E}_{\mathrm{B}}\left(\mathrm{n} T_{s}-\tau\right) \mathrm{S}_{\mathrm{b}}-\mathrm{E}_{\mathrm{C}}\left(\mathrm{n} T_{s}-\tau\right) \mathrm{S}_{\mathrm{c}}\right] e^{\mathrm{j}\left(2 \pi\left(\mathrm{f}_{\mathrm{E} 1}+f_{d}\right) \mathrm{n} T_{s}\right)}+\right.$ $\left.n_{o}\left(\mathrm{n} T_{s}\right)\right)$

After removing the carrier frequency with Doppler shift, i.e. when the locally generated frequency matches the frequency of the orthogonal signal, then the exponential term in equation (8) becomes equal to 1 . The noise component $n_{o}$ is considered uncorrelated, and for the sake of simplicity will be ignored, and then the matched signal is shown in equation (9), which contains the codes and the navigation message only, but in a complex format $\mathrm{X}_{\mathrm{Co}}$. 
$\mathrm{X}_{\mathrm{Co}}\left[n T_{s}\right]=\left[\mathrm{E}_{\mathrm{B}}\left(\mathrm{n} T_{s}-\tau\right) \mathrm{S}_{\mathrm{b}}-\mathrm{E}_{\mathrm{C}}\left(\mathrm{n} T_{s}-\tau\right) \mathrm{S}_{\mathrm{c}}\right]-$

$$
\mathrm{j}\left[\mathrm{E}_{\mathrm{B}}\left(\mathrm{n} T_{s}-\tau\right) \mathrm{S}_{\mathrm{b}}-\mathrm{E}_{\mathrm{C}}\left(\mathrm{n} T_{s}-\tau\right) \mathrm{S}_{\mathrm{c}}\right]
$$

At this stage, we orthogonalise the replica BOC-codes of the data and the pilot channels. Therefore, the primary BOCcode of the data channel will be located in the real part of the equation (10), while the primary BOC-code of the pilot channel will be located in its imaginary part. This represents the complex generated code $\mathrm{C}_{\mathrm{C}}$ and as follows:

$\mathrm{C}_{\mathrm{C}}\left[n T_{s}\right]=\left[\mathrm{E}_{\mathrm{B}}\left(\mathrm{n} T_{s}-\tau\right) \mathrm{S}_{\mathrm{b}}\right]+\mathrm{j}\left[\mathrm{E}_{\mathrm{C}}\left(\mathrm{n} T_{s}-\tau\right) \mathrm{S}_{\mathrm{c}}\right]$

We now multiply this code with the matched signal of equation (9):

$Y_{\mathrm{BC}}=\operatorname{IFFT}\left[F F T\left(\mathrm{X}_{\mathrm{Co}}\left[n T_{s}\right]\right) \cdot F F T\left(\mathrm{C}_{\mathrm{C}}\left[n T_{s}\right]\right)^{*}\right]$

where IFFT represent the inverse Fourier transform FFT is the Fourier transform and $\left(\mathrm{C}_{\mathrm{C}}\left[n T_{s}\right]\right)^{*}$ is the complex conjugate of the complex generated code.

The aligned complex output of this stage is shown in equation (12).

$\mathrm{Y}_{\mathrm{BC}}=\mathrm{ND}_{\mathrm{E} 1 \_\mathrm{B}}-j \mathrm{~N} \mathrm{D}_{\mathrm{E} 1_{\mathrm{B}}}-\mathrm{N} \mathrm{D}_{\mathrm{E} 1_{\mathrm{C}}}-j \mathrm{~N} \mathrm{D}_{\mathrm{E} 1 \_\mathrm{C}}$

where $\mathrm{D}_{\mathrm{E} 1_{\_} \mathrm{B}}$ is the navigation message, $\mathrm{D}_{\mathrm{E} 1_{-} \mathrm{c}}$ is the secondary code and $\mathrm{N}$ is the total number of samples.

Finally, we square this complex output to represent the correlation output, $\mathrm{S}_{\mathrm{BC}-\mathrm{OGSR}}$ as follows:

$S_{B C-O G S R}\left(\tau, f_{d}\right)=2 \mathrm{~N}^{2} \mathrm{D}_{\mathrm{E} 1 \_\mathrm{B}}+2 \mathrm{~N}^{2} \mathrm{D}_{\mathrm{E} 1 \_\mathrm{C}}$

\section{v. Experimental Setup and Results}

Simulink/MATLAB is used to simulate the OGCR implementation, with the sampling frequency of the Galileo signal is set at $16.368 \mathrm{MHz}$, and the folding frequency (IF) at 4.092MHZ. Figure 6 shows a snapshot of the Galileo-OS signal Simulink block diagram and Figure 7 depicted the Galileo-OS signal generation block that is implemented based on equation (5).

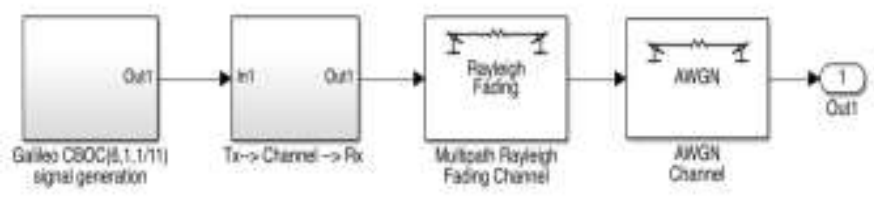

Figure 6. Galileo CBOC $(6,1,1 / 11)$ signal transmitter channel (upper-level block diagram in Simulink)

Various scenarios were chosen to validate the performance of our OGSR implementation, as follows:

1. $(\mathrm{Tx} \rightarrow$ Channel $\rightarrow \mathrm{Rx})$ Block: the received power range from $-127 \mathrm{dBm}$ to $-150 \mathrm{dBm}$.

2. Rayleigh Fading Block: two types of Doppler spectrum are used "Flat and Gaussian" to create multipath signals, which are up to 3 multipath signals in each generated signal.
3. AWGN Block: in this particular block the chosen Mode was SNR-mode to control the amount of the additive white Gaussian noise.

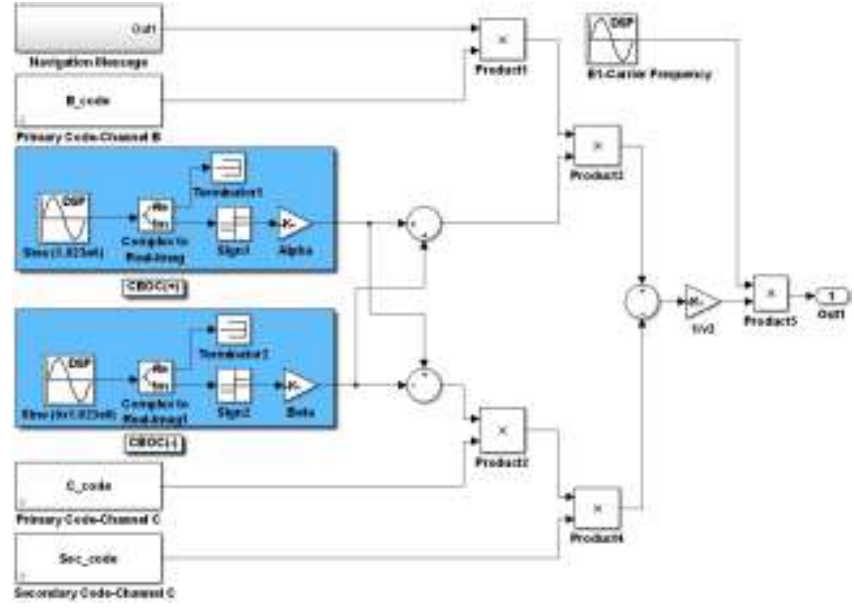

Figure 7. Galileo CBOC $(6,1,1 / 11)$ signal generation

To compare our OGSR with other commonly used methods, we have implemented the DC method that shown in Figure 2. The performance comparison (probability of detection) between the methods is conducted by using FFTsearch algorithm with PRN code length equal to 4092 Chip based on CBOC modulation and Doppler frequency bin equal to $166.6 \mathrm{~Hz}(2 /(3 \mathrm{~T}) \mathrm{Hz}$, where $\mathrm{T}$ is the $4 \mathrm{~ms}$ search dwell time).

As seen in Figure 8, the probability of detection using OGSR and DC are better than using SC (data or pilot) by $2.8 \mathrm{~dB}$. This is achieved as a result of combining the accumulated power of the data and the pilot signals. The results also show that the OGSR's performance is as good as the DC's performance. This is because the correlation output of our OGSR $\left(\mathrm{S}_{\mathrm{BC}-\mathrm{OGSR}}\right)$ is equivalent to the correlation output of the DC method, as described in section IV.

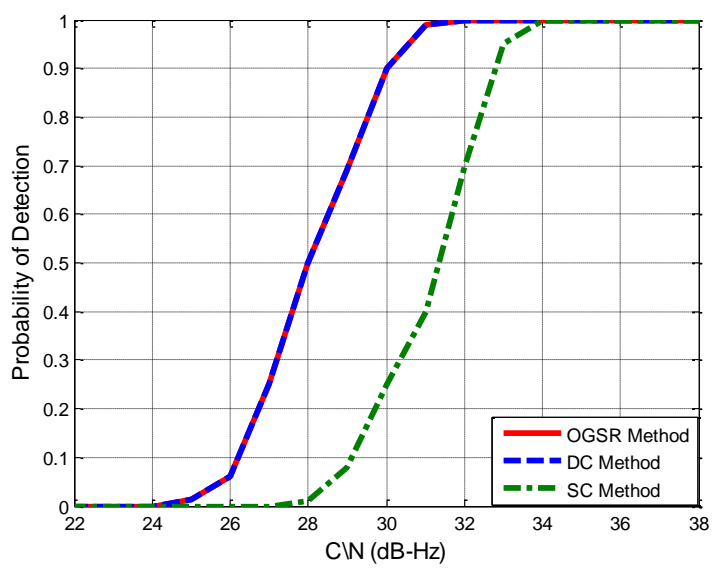

Figure 8. Probability of detection vs. CIN

For the processing time comparison, we performed Monte Carlo simulations with 100 runs to calculate the average processing time. In this comparison, all the methods are run with the same simulation scenario. As shown in Table I, the 
processing time achieved by our OGSR is less than the DC's processing time by $35 \%$.

TABLE I Processing Time

\begin{tabular}{|l|l|}
\hline Method & Processing Time \\
\hline OGSR Method & $6.6306 \mathrm{sec}$. \\
\hline DC Method & $10.1636 \mathrm{sec}$. \\
\hline
\end{tabular}

Finally, Figure 9 illustrates the computational complexity of our OGSR compared with DC implementation. As shown the computational complexity of our OGSR is less by $49 \%$ than the DC implementation.

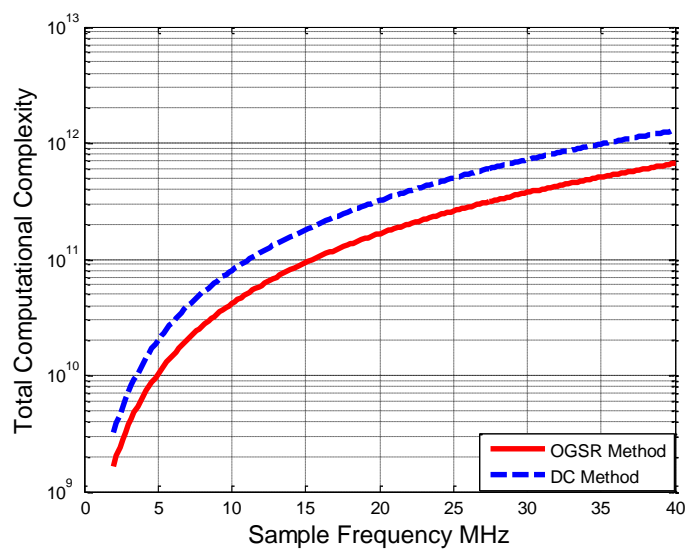

Figure 9. Total computational complexity

\section{vi. Conclusion}

This paper proposes a new joint-data-pilot signal acquisition method for Galileo-OS signal. The novelty of this work focuses on joining and acquiring these data and pilot signals in a single correlation chain by forming them in an orthogonal format. So this process saves valuable resources and accelerates the acquisition time in comparison with conventional joining methods, such as time-domain implementation "4-correlation channels" and frequencydomain implementation "2-correlation channels".

The implementation requirements and detection performance are compared and analysed with the DC acquisition method. The results show that our OGSR performance is as good as DC method because our OGSR combines the Galileo-OS data and pilot signals' powers. In fact the significant reductions are achieved in terms of the computational complexity $(49 \%)$ and the processing time $(35 \%)$; these make our OGSR a good candidate for Smartphone's software receiver.

\section{Acknowledgment}

The researchers would like to thank the Iraqi Ministry of Higher Education for the Ph.D. grant awarded to the first and third authors.

\section{References}

[1] O. ICD, European GNSS (Galileo) open service. Signal in space. Interface control document. Issue 1.1, 2010.

[2] D. Borio and L. Lo Presti, "Data and pilot combining for composite GNSS signal acquisition," International Journal of Navigation and Observation, vol. 2008, 2008.

[3] B. A. Siddiqui, J. Zhang, M. Z. H. Bhuiyan and E. S. Lohan, "Joint datapilot acquisition and tracking of Galileo E1 open service signal," in Ubiquitous Positioning Indoor Navigation and Location Based Service (UPINLBS), 2010, 2010.

[4] T. H. Ta, F. Dovis and L. L. Presti, "A differential joint data/pilot strategy for high sensitivity Galileo E1 signal acquisition," in Advanced Technologies for Communications, 2008. ATC 2008. International Conference on, 2008.

[5] Q. Wan and W. Meng, "Space-Differential Cooperative acquisition for Galileo E1 OS signals," in Computer Science and Information Technology (ICCSIT), 2010 3rd IEEE International Conference on, 2010.

[6] I. A. Lami and M. Ai-Aboodi, "OBPSR: A multi-signal receiver based on the orthogonal and bandpass sampling techniques," in Computer Applications Technology (ICCAT), 2013 International Conference on, 2013.

[7] G. W. Hein, J.-A. Avila-Rodriguez, S. Wallner, A. R. Pratt, J. Owen, J.-L. Issler, J. W. Betz, C. J. Hegarty, L. S. Lenahan, J. J. Rushanan and others, "MBOC: the new optimized spreading modulation recommended for GALILEO L1 OS and GPS L1C," in Proceedings of the IEEE/ION position, location, and navigation symposium, 2006. 